MATEC Web of Conferences 44, 02053 (2016)

DOI: $10.1051 /$ matecconf/20164402053

(C) Owned by the authors, published by EDP Sciences, 2016

\title{
The stability analysis of a rectangular plate supported at points of the corners
}

\author{
Yu Hong Bian ${ }^{a}$, Chen Zhang \\ Key Laboratory of Mechanical Reliability for Heavy Equipments and Large Structures of Hebei Province, College of Civil Engineering and \\ Mechanics, Yanshan University, Qinhuangdao, China
}

\begin{abstract}
The stability problem of a rectangular plate supported at points of corners under uniform pressures in its median plane is studied by using the reciprocal theorem. At first, according to the differential equation of transversal buckling displacement function for a thin rectangular plate, dual trigonometric series, and the reciprocal theorem, the equation of the deflection surface for a rectangular plate with four simple supported edges under uniform pressures in its median plane and a transversal concentrated unit load is established. Secondly, using the reciprocal theorem, the buckling equation for a rectangular plate supported at points of corners is derived. The unknowns here can be determined by the boundary conditions. The exact solution of critical load is given. Finally, as an example, a square plate has been calculated. The results show that the method is accurate and effective to solve the stability problem of rectangular plates. It can serve as technical reference for engineering designs.
\end{abstract}

\section{Introduction}

Applications of thin elastic plate are broad in many fields of aerospace industry, civil engineering, and chemical engineering and so on. However, fulsome pressures imposed upon the edges of plates can cause the breakage because of instability, even causing harm. Therefore, the stability analysis for thin elastic plate is both theoretically and practically an important topic.

Research methods about the stability problem of thin elastic rectangular plates are abundant [1-5]. However, there aren't many studies of the exact solution for the stability problem of rectangular plates with complex boundary conditions. This is because the formulas of the stability problem are generally listed according to the boundary conditions at the stability analysis for the plate. The more complex the boundary condition, the greater the difficulty of solving. In this paper, the reciprocal theorem is applied to research on the stability problem of a rectangular plate supported at points of corners under uniform pressures in its median plane. The exact solution of critical load is given. The results show that the method can solve the stability problem of rectangular plates with various boundary conditions. It is effective to solve the stability problem of thin elastic plates.

\section{Buckling equation for a rectangular plate supported at points of corners}

A rectangular plate with four simple supported edges under uniform pressure $p_{x}$ and $p_{y}$ in its median plane on the boundaries and a transversal concentrated unit load $\delta(x-\xi ; y-\eta)$ at a moving point $(\xi, \eta)$ is shown in Fig. 1, where

$$
\delta(x-\xi ; y-\eta)= \begin{cases}1 & x=\xi, y=\eta \\ 0 & \text { otherlocations }\end{cases}
$$

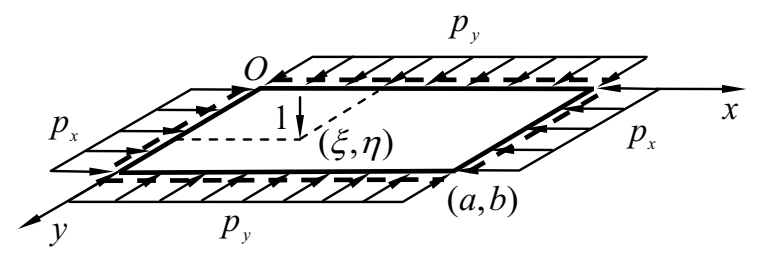

Figure 1. A rectangular plate with four simple supported edges.

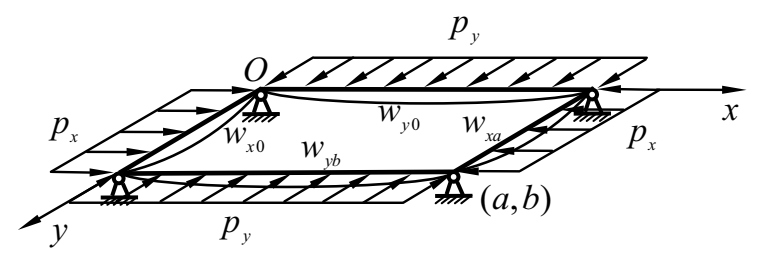

Figure 2. A rectangular plate supported at points of the corners.

The equation of the deflection surface can be easily obtained as

\footnotetext{
${ }^{\mathrm{a}}$ Corresponding author: bianyuhong@163.com
} 


$$
\begin{aligned}
w(x, y ; \xi, \eta)= & \sum_{m=1}^{\infty} \sum_{n=1}^{\infty} \frac{4}{a b D K_{m n}} \sin \frac{m \pi \xi}{a} \sin \frac{n \pi \eta}{b} \\
& \sin \frac{m \pi x}{a} \sin \frac{n \pi y}{b}
\end{aligned}
$$

where $D$ is the bending rigidity of the rectangular plate, and

$$
K_{m n}=\left(\frac{m^{2} \pi^{2}}{a^{2}}+\frac{n^{2} \pi^{2}}{b^{2}}\right)^{2}-\frac{p_{x}}{D} \frac{m^{2} \pi^{2}}{a^{2}}-\frac{p_{y}}{D} \frac{n^{2} \pi^{2}}{b^{2}}
$$

Considering a rectangular plate supported at points of the corners shown in Fig. 2, the plate is in a critical condition under the pressure $p_{x}$ and $p_{y}$ in its median plane. The deflection equations of four free edges are respectively assumed as

$$
\begin{aligned}
& w_{x 0}=\sum_{n=1}^{\infty} a_{n} \sin \frac{n \pi y}{b} \\
& w_{x a}=\sum_{n=1}^{\infty} b_{n} \sin \frac{n \pi y}{b} \\
& w_{y 0}=\sum_{m=1}^{\infty} c_{m} \sin \frac{m \pi x}{a} \\
& w_{y b}=\sum_{m=1}^{\infty} d_{m} \sin \frac{m \pi x}{a}
\end{aligned}
$$

The rectangular plate with four simple supported edges shown in Fig. 1 is taken as the first system; the rectangular plate supported at points of corners shown in Fig. 2 is taken as the second system. Without regard to manual action, between the first system and the second system, the reciprocal theorem is applied, there are

$$
\begin{gathered}
w(\xi, \eta)=\int_{0}^{b} V_{1 x 0} w_{x 0} \mathrm{~d} y-\int_{0}^{b} V_{1 x a} w_{x a} \mathrm{~d} y+ \\
\int_{0}^{a} V_{1 y 0} w_{y 0} \mathrm{~d} x-\int_{0}^{a} V_{1 y b} w_{y b} \mathrm{~d} x
\end{gathered}
$$

where $V_{1 x 0}, V_{1 x a}, V_{1 y 0}$, and $V_{1 y b}$ are equivalent shearing forces on boundaries for the first system.

The Eqs. (2)-(7) are substituted into the Eq. (8), the buckling equation for a rectangular plate supported at points of corners can be obtained as

$$
\begin{gathered}
w(\xi, \eta)=\sum_{n=1}^{\infty} \frac{1}{\alpha_{n}^{2}-\beta_{n}^{2}}\left\{\left[\alpha_{n}^{2}-(2-v)\left(\frac{n \pi}{b}\right)^{2}+\right.\right. \\
\left.\frac{p_{x}}{D}\right] \frac{\operatorname{sh} \alpha_{n}(a-\xi)}{\operatorname{sh} \alpha_{n} a}-\left[\beta_{n}^{2}-(2-v)\left(\frac{n \pi}{b}\right)^{2}+\right. \\
\left.\left.\frac{p_{x}}{D}\right] \frac{\operatorname{sh} \beta_{n}(a-\xi)}{\operatorname{sh} \beta_{n} a}\right\} \sin \frac{n \pi \eta}{b}\left(a_{n}\right)+
\end{gathered}
$$

$$
\begin{aligned}
& \sum_{n=1}^{\infty} \frac{1}{\alpha_{n}^{2}-\beta_{n}^{2}}\left\{\left[\alpha_{n}^{2}-(2-v)\left(\frac{n \pi}{b}\right)^{2}+\frac{p_{x}}{D}\right] \frac{\operatorname{sh} \alpha_{n} \xi}{\operatorname{sh} \alpha_{n} a}-\right. \\
& \left.\left[\beta_{n}^{2}-(2-v)\left(\frac{n \pi}{b}\right)^{2}+\frac{p_{x}}{D}\right] \frac{\operatorname{sh} \beta_{n} \xi}{\operatorname{sh} \beta_{n} a}\right\} \sin \frac{n \pi \eta}{b}\left(b_{n}\right)+ \\
& \sum_{m=1}^{\infty} \frac{1}{\alpha_{m}^{2}-\beta_{m}^{2}}\left\{\left[\alpha_{m}^{2}-(2-v)\left(\frac{m \pi}{a}\right)^{2}+\right.\right. \\
& \left.\frac{p_{y}}{D}\right] \frac{\operatorname{sh} \alpha_{m}(b-\eta)}{\operatorname{sh} \alpha_{m} b}-\left[\beta_{m}^{2}-(2-v)\left(\frac{m \pi}{a}\right)^{2}+\right. \\
& \left.\left.\frac{p_{y}}{D}\right] \frac{\operatorname{sh} \beta_{m}(b-\eta)}{\operatorname{sh} \beta_{m} b}\right\} \sin \frac{m \pi \xi}{a}\left(c_{m}\right)+ \\
& \sum_{m=1}^{\infty} \frac{1}{\alpha_{m}^{2}-\beta_{m}^{2}}\left\{\left[\alpha_{m}^{2}-(2-v)\left(\frac{m \pi}{a}\right)^{2}+\frac{p_{y}}{D}\right] \frac{\operatorname{sh} \alpha_{m} \eta}{\operatorname{sh} \alpha_{m} b}-\right. \\
& \left.\left[\beta_{m}^{2}-(2-v)\left(\frac{m \pi}{a}\right)^{2}+\frac{p_{y}}{D}\right] \frac{\operatorname{sh} \beta_{m} \eta}{\operatorname{sh} \beta_{m} b}\right\} \sin \frac{m \pi \xi}{a}\left(d_{m}\right)
\end{aligned}
$$

where $v$ is Poisson's ratio of the plate, and

$$
\begin{aligned}
& \alpha_{n}=\sqrt{\eta_{n}+\sqrt{\eta_{n}^{2}-p_{n}^{2}}}, \beta_{n}=\sqrt{\eta_{n}-\sqrt{\eta_{n}^{2}-p_{n}^{2}}} \\
& \eta_{n}=\left(\frac{n \pi}{b}\right)^{2}-\frac{p_{x}}{2 D}, p_{n}^{2}=\left(\frac{n \pi}{b}\right)^{4}-\frac{p_{y}}{D}\left(\frac{n \pi}{b}\right)^{2} \\
& \alpha_{m}=\sqrt{\eta_{m}+\sqrt{\eta_{m}^{2}-p_{m}^{2}}}, \beta_{m}=\sqrt{\eta_{m}-\sqrt{\eta_{m}^{2}-p_{m}^{2}}} \\
& \eta_{m}=\left(\frac{m \pi}{a}\right)^{2}-\frac{p_{y}}{2 D}, p_{m}^{2}=\left(\frac{m \pi}{a}\right)^{4}-\frac{p_{x}}{D}\left(\frac{m \pi}{a}\right)^{2}
\end{aligned}
$$

\section{Characteristic equation of critical load}

The Eq. (9) should satisfy the following boundary conditions:

$$
\begin{aligned}
& \left\{-D\left[\frac{\partial^{3} w}{\partial \xi^{3}}+(2-v) \frac{\partial^{3} w}{\partial \xi \partial \eta^{2}}\right]-p_{x} \frac{\partial w}{\partial \xi}\right\}_{\xi=0}=0 \\
& \left\{-D\left[\frac{\partial^{3} w}{\partial \xi^{3}}+(2-v) \frac{\partial^{3} w}{\partial \xi \partial \eta^{2}}\right]-p_{x} \frac{\partial w}{\partial \xi}\right\}_{\xi=a}=0 \\
& \left\{-D\left[\frac{\partial^{3} w}{\partial \eta^{3}}+(2-v) \frac{\partial^{3} w}{\partial \eta \partial \xi^{2}}\right]-p_{y} \frac{\partial w}{\partial \eta}\right\}_{\eta=0}=0
\end{aligned}
$$




$$
\left\{-D\left[\frac{\partial^{3} w}{\partial \eta^{3}}+(2-v) \frac{\partial^{3} w}{\partial \eta \partial \xi^{2}}\right]-p_{y} \frac{\partial w}{\partial \eta}\right\}_{\eta=b}=0
$$

The Eq. (9) is substituted into the Eqs. (14)-(17), after simplified and arranged, the executing equations for above boundary conditions can be respectively obtained as

$$
\begin{gathered}
\frac{1}{\alpha_{n}^{2}-\beta_{n}^{2}}\left\{\left[\alpha_{n}^{2}-(2-v) \frac{n^{2} \pi^{2}}{b^{2}}+\frac{p_{x}}{D}\right]^{2} \alpha_{n} \operatorname{cth} \alpha_{n} a-\right. \\
\left.\left[\beta_{n}^{2}-(2-v) \frac{n^{2} \pi^{2}}{b^{2}}+\frac{p_{x}}{D}\right]^{2} \beta_{n} \operatorname{cth} \beta_{n} a\right\}\left(a_{n}\right)- \\
\frac{1}{\alpha_{n}^{2}-\beta_{n}^{2}}\left\{\left[\alpha_{n}^{2}-(2-v) \frac{n^{2} \pi^{2}}{b^{2}}+\frac{p_{x}}{D}\right]^{2} \frac{\alpha_{n}}{\operatorname{sh} \alpha_{n} a}-\right. \\
\left.\left[\beta_{n}^{2}-(2-v) \frac{n^{2} \pi^{2}}{b^{2}}+\frac{p_{x}}{D}\right]^{2} \frac{\beta_{n}}{\operatorname{sh} \beta_{n} a}\right\}\left(b_{n}\right)+ \\
\frac{2}{b} \sum_{m=1}^{\infty} \frac{1}{K_{m n}} \frac{m \pi}{a} \frac{n \pi}{b}\left[(1-v)^{2} \frac{m^{2} \pi^{2}}{a^{2}} \frac{n^{2} \pi^{2}}{b^{2}}-\right. \\
\left.\frac{p_{y}}{D} \frac{m^{2} \pi^{2}}{a^{2}}-\frac{p_{x}}{D} \frac{n^{2} \pi^{2}}{b^{2}}+\frac{p_{x} p_{y}}{D^{2}}\right]\left(c_{m}\right)- \\
\frac{2}{b} \sum_{m=1}^{\infty} \frac{(-1)^{n}}{K_{m n}} \frac{m \pi}{a} \frac{n \pi}{b}\left[(1-v)^{2} \frac{m^{2} \pi^{2}}{a^{2}} \frac{n^{2} \pi^{2}}{b^{2}}-\right. \\
\left.\frac{p_{y}}{D} \frac{m^{2} \pi^{2}}{a^{2}}-\frac{p_{x}}{D} \frac{n^{2} \pi^{2}}{b^{2}}+\frac{p_{x} p_{y}}{D^{2}}\right]\left(d_{m}\right)=0
\end{gathered}
$$$$
\frac{1}{\alpha_{n}^{2}-\beta_{n}^{2}}\left\{\left[\alpha_{n}^{2}-(2-v) \frac{n^{2} \pi^{2}}{b^{2}}+\frac{p_{x}}{D}\right]^{2} \frac{\alpha_{n}}{\operatorname{sh} \alpha_{n} a}-\right.
$$$$
\left.\left[\beta_{n}^{2}-(2-v) \frac{n^{2} \pi^{2}}{b^{2}}+\frac{p_{x}}{D}\right]^{2} \frac{\beta_{n}}{\operatorname{sh} \beta_{n} a}\right\}\left(a_{n}\right)-
$$$$
\frac{1}{\alpha_{n}^{2}-\beta_{n}^{2}}\left\{\left[\alpha_{n}^{2}-(2-v) \frac{n^{2} \pi^{2}}{b^{2}}+\frac{p_{x}}{D}\right]^{2} \alpha_{n} \operatorname{cth} \alpha_{n} a-\right.
$$$$
\left.\left[\beta_{n}^{2}-(2-v) \frac{n^{2} \pi^{2}}{b^{2}}+\frac{p_{x}}{D}\right]^{2} \beta_{n} \operatorname{cth} \beta_{n} a\right\}\left(b_{n}\right)+
$$$$
\frac{2}{b} \sum_{m=1}^{\infty} \frac{(-1)^{m}}{K_{m n}} \frac{m \pi}{a} \frac{n \pi}{b}\left[(1-v)^{2} \frac{m^{2} \pi^{2}}{a^{2}} \frac{n^{2} \pi^{2}}{b^{2}}-\right.
$$$$
\left.\frac{p_{y}}{D} \frac{m^{2} \pi^{2}}{a^{2}}-\frac{p_{x}}{D} \frac{n^{2} \pi^{2}}{b^{2}}+\frac{p_{x} p_{y}}{D^{2}}\right]\left(c_{m}\right)-
$$$$
\frac{2}{b} \sum_{m=1}^{\infty} \frac{(-1)^{m+n}}{K_{m n}} \frac{m \pi}{a} \frac{n \pi}{b}\left[(1-v)^{2} \frac{m^{2} \pi^{2}}{a^{2}} \frac{n^{2} \pi^{2}}{b^{2}}-\right.
$$

$$
\left.\frac{p_{y}}{D} \frac{m^{2} \pi^{2}}{a^{2}}-\frac{p_{x}}{D} \frac{n^{2} \pi^{2}}{b^{2}}+\frac{p_{x} p_{y}}{D^{2}}\right]\left(d_{m}\right)=0
$$

$$
\begin{aligned}
& \frac{2}{a} \sum_{n=1}^{\infty} \frac{1}{K_{m n}} \frac{m \pi}{a} \frac{n \pi}{b}\left[(1-v)^{2} \frac{m^{2} \pi^{2}}{a^{2}} \frac{n^{2} \pi^{2}}{b^{2}}-\right. \\
& \left.\frac{p_{y}}{D} \frac{m^{2} \pi^{2}}{a^{2}}-\frac{p_{x}}{D} \frac{n^{2} \pi^{2}}{b^{2}}+\frac{p_{x} p_{y}}{D^{2}}\right]\left(a_{n}\right)- \\
& \frac{2}{a} \sum_{n=1}^{\infty} \frac{(-1)^{m}}{K_{m n}} \frac{m \pi}{a} \frac{n \pi}{b}\left[(1-v)^{2} \frac{m^{2} \pi^{2}}{a^{2}} \frac{n^{2} \pi^{2}}{b^{2}}-\right. \\
& \left.\frac{p_{y}}{D} \frac{m^{2} \pi^{2}}{a^{2}}-\frac{p_{x}}{D} \frac{n^{2} \pi^{2}}{b^{2}}+\frac{p_{x} p_{y}}{D^{2}}\right]\left(b_{n}\right)+ \\
& \frac{1}{\alpha_{m}^{2}-\beta_{m}^{2}}\left\{\left[\alpha_{m}^{2}-(2-v) \frac{m^{2} \pi^{2}}{a^{2}}+\frac{p_{y}}{D}\right]^{2} \alpha_{m} \operatorname{cth} \alpha_{m} b-\right. \\
& \left.\left[\beta_{m}^{2}-(2-v) \frac{m^{2} \pi^{2}}{a^{2}}+\frac{p_{y}}{D}\right]^{2} \beta_{m} \operatorname{cth} \beta_{m} b\right\}\left(c_{m}\right)- \\
& \frac{1}{\alpha_{m}^{2}-\beta_{m}^{2}}\left\{\left[\alpha_{m}^{2}-(2-v) \frac{m^{2} \pi^{2}}{a^{2}}+\frac{p_{y}}{D}\right]^{2} \frac{\alpha_{m}}{\operatorname{sh} \alpha_{m} b}-\right. \\
& \left.\left[\beta_{m}^{2}-(2-v) \frac{m^{2} \pi^{2}}{a^{2}}+\frac{p_{y}}{D}\right]^{2} \frac{\beta_{m}}{\operatorname{sh} \beta_{m} b}\right\}\left(d_{m}\right)=0 \\
& \frac{2}{a} \sum_{n=1}^{\infty} \frac{(-1)^{n}}{K_{m n}} \frac{m \pi}{a} \frac{n \pi}{b}\left[(1-v)^{2} \frac{m^{2} \pi^{2}}{a^{2}} \frac{n^{2} \pi^{2}}{b^{2}}-\right. \\
& \left.\frac{p_{y}}{D} \frac{m^{2} \pi^{2}}{a^{2}}-\frac{p_{x}}{D} \frac{n^{2} \pi^{2}}{b^{2}}+\frac{p_{x} p_{y}}{D^{2}}\right]\left(a_{n}\right)- \\
& \frac{2}{a} \sum_{n=1}^{\infty} \frac{(-1)^{m+n}}{K_{m n}} \frac{m \pi}{a} \frac{n \pi}{b}\left[(1-v)^{2} \frac{m^{2} \pi^{2}}{a^{2}} \frac{n^{2} \pi^{2}}{b^{2}}-\right. \\
& \left.\frac{p_{y}}{D} \frac{m^{2} \pi^{2}}{a^{2}}-\frac{p_{x}}{D} \frac{n^{2} \pi^{2}}{b^{2}}+\frac{p_{x} p_{y}}{D^{2}}\right]\left(b_{n}\right)+ \\
& \frac{1}{\alpha_{m}^{2}-\beta_{m}^{2}}\left\{\left[\alpha_{m}^{2}-(2-v) \frac{m^{2} \pi^{2}}{a^{2}}+\frac{p_{y}}{D}\right]^{2} \frac{\alpha_{m}}{\operatorname{sh} \alpha_{m} b}-\right. \\
& \left.\left[\beta_{m}^{2}-(2-v) \frac{m^{2} \pi^{2}}{a^{2}}+\frac{p_{y}}{D}\right]^{2} \frac{\beta_{m}}{\operatorname{sh} \beta_{m} b}\right\}\left(c_{m}\right)- \\
& \frac{1}{\alpha_{m}^{2}-\beta_{m}^{2}}\left\{\left[\alpha_{m}^{2}-(2-v) \frac{m^{2} \pi^{2}}{a^{2}}+\frac{p_{y}}{D}\right]^{2} \alpha_{m} \operatorname{cth} \alpha_{m} b-\right. \\
& \left.\left[\beta_{m}^{2}-(2-v) \frac{m^{2} \pi^{2}}{a^{2}}+\frac{p_{y}}{D}\right]^{2} \beta_{m} \operatorname{cth} \beta_{m} b\right\}\left(d_{m}\right)=0
\end{aligned}
$$


The Eqs. (18)-(21) form a set of the homogeneous linear equations, which include the unknowns $a_{n}, b_{n}$, $c_{m}$, and $d_{m}$ in all. Setting the determinant of the coefficients equal to zero is the characteristic equation for the stability problem.

\section{Numerical example}

The buckling surface of the plate is symmetrical or antisymmetric about $x=a / 2$ and $y=b / 2$. Four forms of the instability may exist by $a_{n}=b_{n}, a_{n}=-b_{n}$, $c_{m}=d_{m}$, and $c_{m}=-d_{m}$. Because symmetrical wave form corresponds to the minimum of critical load, it is reasonable to think about the Eq. (18) and (20) only. A square plate is used as an example. Let $a=b, v=0.3$, $p_{x}=p_{y}=p$. The $p_{c r}=p a^{2} / D \pi^{2}$ is introduced as the coefficient of critical load. The Eqs. (10)-(13) are respectively substituted into the Eq. (18) and (20), there are

$$
\begin{aligned}
& \frac{1}{p_{c r}}\left\{\left[p_{c r}-(1-v) n^{2}\right]^{2} n \pi \text { th } \frac{n \pi}{2}-\right. \\
& \left.(1-v)^{2} n^{4} \sqrt{n^{2}-p_{c r}} \pi \operatorname{th} \frac{\sqrt{n^{2}-p_{c r}}}{2} \pi\right\}\left(a_{n}\right)+ \\
& \sum_{m=1}^{\infty} \frac{4\left[n^{2}+(2-v) m^{2}-p_{c r}\right]}{m n\left[\left(\frac{m}{n}+\frac{n}{m}\right)^{2}-\left(\frac{1}{m^{2}}+\frac{1}{n^{2}}\right) p_{c r}\right]} \\
& {\left[m^{2}+(2-v) n^{2}-p_{c r}\right]\left(c_{m}\right)=0} \\
& \frac{1}{p_{c r}}\left\{\left[p_{c r}-(1-v) m^{2}\right]^{2} m \pi \operatorname{th} \frac{m \pi}{2}-\right. \\
& \left.(1-v)^{2} m^{4} \sqrt{m^{2}-p_{c r}} \pi \operatorname{th} \frac{\sqrt{m^{2}-p_{c r}}}{2} \pi\right\}\left(c_{m}\right)+ \\
& \sum_{n=1}^{\infty} \frac{4\left[m^{2}+(2-v) n^{2}-p_{c r}\right]}{m n\left[\left(\frac{n}{m}+\frac{m}{n}\right)^{2}-\left(\frac{1}{m^{2}}+\frac{1}{n^{2}}\right) p_{c r}\right]} . \\
& {\left[n^{2}+(2-v) m^{2}-p_{c r}\right]\left(a_{n}\right)=0}
\end{aligned}
$$

The corresponding critical load can be obtained by the determinant of the coefficients for above homogeneous linear equations equal to zero. Considering the symmetry of the plate, let $m=1,3,5, \cdots, n=1,3,5, \cdots$. Using the method of progressive searching root and programming the characteristic equation of the Eq. (22) and (23), the results are shown in Table 1.
Table 1. Critical load coefficients $p_{c r}$ of the rectangular plate supported at points of corners.

\begin{tabular}{|c|c|c|c|c|}
\hline$k$ & 8 & 12 & 16 & 20 \\
\hline$p_{c r}$ & 1.4079 & 1.3268 & 1.3004 & 1.2589 \\
\hline \hline$k$ & 24 & 32 & 50 & 64 \\
\hline$p_{c r}$ & 1.2177 & 1.1826 & 1.1168 & 1.1151 \\
\hline
\end{tabular}

where $k$ is ranks of the determinant, i.e. the sums of the $m$ and $n$. The $p_{c r}=1.1151$ can be selected as the exact solution of critical load for the rectangular plate supported at points of corners.

\section{Conclusions}

By analyzing the stability of a rectangular plate supported at points of corners under uniform pressures in its median plane, the exact solution of critical load is given. The results show that the method is accurate and effective to solve the stability problem of thin elastic plates. The results of this paper will overcome errors of various approximate solutions. It provides reliable basis for the security and reliability design of engineering structures.

\section{References}

1. I. E. Harik, R. Ekambaram, Seminumerical solution for buckling of rectangular plates, Computers and Structures, 23, 649-655 (1986)

2. F. F. Zhang, Elastic thin plates, 2nd ed, Beijing: Science Press (1984)

3. J. Purbolaksono, M. H. Aliabadi, Buckling analysis of shear deformable plates by boundary element method, International Journal for Numerical Methods in Engineering, 62, 537-563 (2005)

4. C. W. Bert, K. K. Devarakonda, Buckling of rectangular plates subjected to nonlinearly distributed in-plane loading, Int. J. Solids Struct., 40, 4097-4106 (2003)

5. X. W. Wang, X. F. Wang, X. D. Shi, Accurate buckling loads of thin rectangular plates under parabolic edge compressions by the differential quadrature method, International Journal of Mechanical Sciences, 49, 447-453 (2007)

6. M. L. Tan, J. L. Dong, Buckling analysis of thin rectangular plates under cosine-distributed compressive loads, Engineering Mechanics, 27, 3235 (2010) 